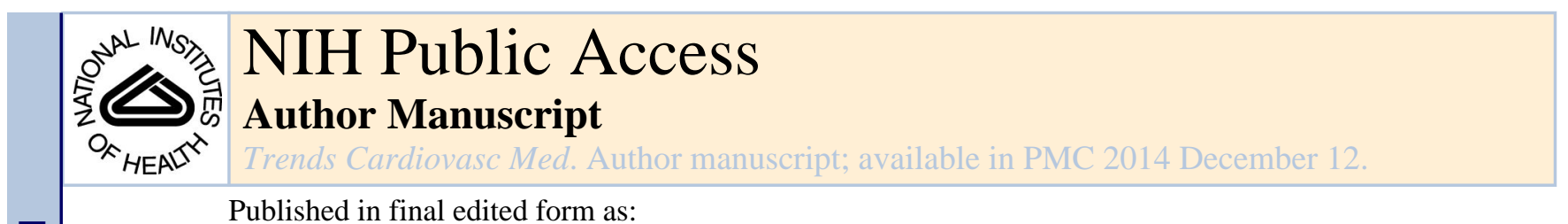

Published in final edited form as:

Trends Cardiovasc Med. 2013 October ; 23(7): 250-256. doi:10.1016/j.tcm.2013.02.001.

\title{
Compartmentalization of $\beta$-Adrenergic Signals in Cardiomyocytes
}

\author{
Qin Fu ${ }^{1,2}$, Xiongwen Chen $^{3}$, and Yang K. Xiang ${ }^{1,}$ \\ ${ }^{1}$ The Department of Pharmacology, University of California at Davis, Davis, CA 95616 \\ ${ }^{2}$ The Department of Pharmacology, Tongji Medical College, Huazhong University of Science and \\ Technology, Wuhan, Hubei 430030, China \\ ${ }^{3}$ The Department of Physiology and Cardiovascular Research Center, Temple University Medical \\ Center, Philadelphia, PA 19140
}

\section{Abstract}

Under $\beta$-adrenergic stimulation, the distribution of cAMP is highly restricted at distinct intracellular domains for compartmentalized activation of protein kinase $\mathrm{A}$, which promotes selective phosphorylation of proteins for contractile responses in cardiomyocytes. This is primarily due to a concert effort between restrictions of cAMP distribution by a family of phosphodiesterases and locally anchored protein kinase A by a family of scaffold A kinaseanchoring proteins. Moreover, these regulatory mechanisms underlie cross talk between $\beta$ adrenergic signals and other receptor stimulated signaling cascades, which alters the compartmentalized $\beta$ adrenergic signals for proper contractility in myocardium. Maintaining integrity of compartmentalized $\beta$-adrenergic signals is critical for physiological cardiac function and for preventing development of cardiac diseases.

\section{Introduction}

Sympathetic activities lead to release of catecholamines from the nerve termini, which activate adrenergic receptors (ARs) on cardiomyocytes to increase cardiac contractile force and rate. Activation of $\beta$ ARs induces intracellular signaling cascades through $\mathrm{G}$ protein and effectors such as adenylyl cyclases (ACs), a family of enzymes that produce second messenger cyclic adenosine monophosphate (cAMP). cAMP activates protein kinase A (PKA) anchored by A kinase anchoring proteins (AKAPs) for substrate phosphorylation. The phosphorylated proteins exert a concert effort to promote contractile force and rate. After the first evidence suggests the notion that the $\beta A R$ subtypes induce compartmentalized cAMP and PKA-mediated target phosphorylation in cardiomyocytes (Xiao et al., 1994), the

\footnotetext{
(C) 2013 Elsevier Inc. All rights reserved.

*Address correspondence to: Yang K. Xiang, PhD, The Department of Pharmacology, University of California at Davis, Davis, CA 95616. Tel 530-752-6895; ykxiang@ucdavis.edu.

Publisher's Disclaimer: This is a PDF file of an unedited manuscript that has been accepted for publication. As a service to our customers we are providing this early version of the manuscript. The manuscript will undergo copyediting, typesetting, and review of the resulting proof before it is published in its final citable form. Please note that during the production process errors may be discovered which could affect the content, and all legal disclaimers that apply to the journal pertain.
} 
later development of genetically coded biosensor allows direct visualization of compartmentalized cAMP in living rat neonatal cardiomyocytes (Zaccolo and Pozzan, 2002), which opens a new era of studies to understand the mechanism and functional implication of localized $\beta$ AR signals. The compartmentalized $\beta$-adrenergic signals in highly differentiated cardiomyocytes raise cAMP and PKA activities at precise subcellular organelles rather than throughout the cell (Xiang, 2011; Zaccolo, 2006). The spatially raised intracellular PKA activities promote phosphorylation of a specific set of substrates in cardiomyocytes for increasing cardiac contractility during physiological responses. Spillover of cAMP for unwanted PKA activities or signals at other cellular domains can contribute to dysfunction of cardiomyocytes, which may subsequently lead to cardiac hypertrophy and apoptosis, and onset of heart failure (Lefkowitz, 2007).

\section{Tonic local CAMP activities under spontaneous $\beta$-adrenergic stimulation}

Under resting physiological conditions, cAMP signals are induced by intrinsic spontaneous $\beta A R$ activities or by baseline levels of catecholamines. Spontaneous activation of $\beta A R s$ leads to activation of both AC5 and AC6, in which AC5 appears to make primary contribution to cAMP synthesis involved in maintaining tonic cAMP activities (Okumura et al., 2003). However, cAMP can be rapidly hydrolyzed by PDEs. Emerging evidence indicates that PDEs play a major role in directing intracellular cAMP propagation (Xiang, 2011; Zaccolo, 2006).

In cardiomyocytes, PDE3 and 4 are the major families expressed together with other PDEs including PDE2, 5 and 8 (Zaccolo, 2006). PDE3 and 4 account for $90 \%$ of the total PDE enzymatic activities in rodent cardiomyocytes, and much less $30 \%$ of activities in human hearts (Richter et al., 2011). These two PDE families have distinct roles in regulating $\beta$ adrenergic signals (Soto et al., 2009) and contraction responses (Xiang et al., 2005). PDE4D isoforms, in particular, are physically associated with $\beta \mathrm{AR} / \mathrm{Gs} / \mathrm{AC}$ complexes, and readily hydrolyze cAMP produced by AC. In mouse cardiomyocytes, $\beta_{2} \mathrm{AR}$ primarily binds to PDE4D9 (De Arcangelis et al., 2009) whereas $\beta_{1}$ AR primarily binds to PDE4D8 (De Arcangelis et al., 2008; Richter et al., 2008). Meanwhile, the organization of $\beta$ AR-ACAKAP complex promotes local PKA activation, which can phosphorylate and activate the receptor-associated PDE4Ds for cAMP degradation (Sette and Conti, 1996), facilitating a negative feedback to confine cAMP signals within the local receptor signaling domains (Mongillo et al., 2004). The balance between cAMP production and cAMP degradation shapes an equilibrium that produces local tonic cAMP activities along the sarcolemma in cardiomyocytes (De Arcangelis et al., 2010). One possible physiological effect of these local tonic cAMP activities is to modulate pacemaker activities and heart rates. Breaking up the equilibrium with inhibition of PDE4 enhances tonic cAMP activities in cardiomyocytes (De Arcangelis et al., 2010). Recently, it is reported that multidrug resistant protein 4 (MRP4) emerges as another important player for clearing up cAMP in cardiomyocytes. MRP4 is a member of the MRP subfamily of ATP-binding cassette transporters (ABCC), which has a remarkable ability to transport signaling molecules such as cAMP (Russel et al., 2008). Inhibition or deletion of MRP4 enhances cAMP signals for PKA phosphorylation and contractile responses under $\beta A R$ stimulation (Sellers et al., 2012). The activated PKA under inhibition of MRP4 also promotes phosphorylation and activation of PDE4, which in turn 
decreases cAMP signals in cardiomyocytes. Together, AC5, MRP4, PDE4, and potential additional regulators coordinate the maintenance of tonic cAMP activities in cardiomyocytes.

\section{Local effects of cAMP signals in the vicinity of $\beta$ ARs on the PM}

Meanwhile, acute stimulation of mouse cardiomyocytes with minimal $1 \mathrm{nM}$ of $\beta \mathrm{AR}$ agonist induces a transient increase of cAMP that rapidly returns to the baseline level (De Arcangelis et al., 2010) due to the negative feedback by PKA-dependent activation of PDE4. This new baseline level represents a shifted and active equilibrium, in which both cAMP production and cAMP degradation are enhanced. Simultaneous stimulation of $\beta$ ARs with 1 $\mathrm{nM}$ catecholamines and inhibition of PDE4 can yield elevated and sustained cAMP signals similar to those under maximal $\beta$ AR stimulation (De Arcangelis et al., 2010), suggesting that minimal stimulation of $\beta$ ARs has the potential to fully promote myocardium contractile machinery.

As one of the key effectors of the raising levels of intracellular cAMP, PKA is anchored to the different subcellular organelles to specific target the local substrates. $\beta$ adrenergic stimulation activates PKA for phosphorylation of substrates such as ion channels including sarcolemmal voltage-gated L-type $\mathrm{Ca}^{2+}$ channels (LTCCs), ryanodine receptors (RyRs) and cardiac sarcoendoplasmic reticular $\mathrm{Ca}^{2+}$-adenosine triphosphatase type 2 (SERCA2) in the SR involved in calcium cycling. To precisely control $\beta$ AR-induced channel activation, PKA are anchored onto ion channels via different AKAPs (Dodge-Kafka et al., 2010; Willoughby et al., 2006), some of which are also in direct association with $\beta$ ARs together with regulatory enzymes including phosphatases (PPs) and phosphodiesterases (PDEs) (Dodge-Kafka et al., 2010; Willoughby et al., 2006). Under spontaneous or minimal adrenergic stimulation, cAMP signals are sufficient to activate PKA and to promote phosphorylation of proteins in the vicinities of receptor complexes (De Arcangelis et al., 2010), which include LTCCs in ventricle myocytes (Balijepalli et al., 2006) and HCN in sinotal atrial cells (Barbuti et al., 2007) as well as AKAPs, PDEs, and PPs. The assembling of these complexes ensures the confinement of cAMP signals in local microdomains to promote PKA actions (Figure 1A). While these confined cAMP/PKA signals are involved in modulating the membrane depolarization and contraction rate, it has minimal effect on contractility that is also dependent on PKA activities on the SR in cardiomyocytes.

Among $\beta$ ARs, the $\beta_{1} \mathrm{AR}$-induced cAMP signals under maximal dose of stimulation are broadly distributed in cardiomyocytes (Nikolaev et al., 2006), which can activate PKA phosphorylation for LTCCs in both local domains and globally (Xiao et al., 1994). In contrast, $\beta_{2} \mathrm{AR}$ associates with a subpopulation of LTCCs localized in caveoli. Maximal dose of stimulation on $\beta_{2}$ AR leads to a small local cAMP signal that is sufficient for PKA activation to phosphorylate LTCCs in the same compartment, which is also modulated by coupling of $\beta_{2} \mathrm{AR}$ to $\mathrm{Gi}$ (Xiao et al., 1994). While the coupling of $\beta_{2} \mathrm{AR}$ to Gi reduces the AC-dependent cAMP production, the agonist-induced recruitment of PDE4D isoforms to the receptor enhances the cAMP degradation (De Arcangelis et al., 2009). Both mechanisms could play roles in reduce cAMP production under excessive $\beta A R$ agonist stimulation, and independently contribute to restriction of cAMP at the local domains. Disruption of lipid 
rafts/caveoli amplifies the $\beta_{2} \mathrm{AR}$-induced cAMP signals, which promote PKA phosphorylation-mediated increase in calcium influx through LTCCs (Balijepalli et al., 2006) and PKA phosphorylation of phospholamban (PLB) on the SR for calcium cycling (Macdougall et al., 2012). Moreover, studies have also identified that AKAP5 (also as AKAP79/150) holds the receptor and AC in caveoli, and controls a $\beta A R$-stimulated microdomain of cAMP that promotes PKA phosphorylation of caveolin-associated LTCCs and $\mathrm{Ca}^{2+}$ transients (Nichols et al., 2010). Meanwhile, the binding of AKAP5 to $\beta_{2} \mathrm{AR}$ also promotes activation of mitogen-activated protein (MAP) kinase cascade whereas the binding of AKAP12 (also as gravin or AKAP250) to $\beta_{2} \mathrm{AR}$ promotes receptor internalization and resensitization (Tao and Malbon, 2008). The significance of organizing $\beta A R$ subtypes into different signal domains by AKAPs in myocardial contractile responses is not well understood. The activation of MAP kinase cascade may contribute to cardiac remodeling in chronic stress and heart disease.

\section{Compartmentalized intracellular cAMP signal propagation under adrenergic stimulation}

Eventually, $\beta$ AR-induced cAMP signals have to propagate to distinct intracellular locations in cardiomyocytes for promoting protein phosphorylation and contractile responses. Using subcellular organelle-anchored biosensors to measure cAMP and PKA activities in live cardiomyocytes, studies suggest that cAMP signals at the PM are functionally segregated and distinct from cAMP signals at the SR network under $\beta A R$ stimulation. For example, stimulation of $\beta A R$ with minimal doses of agonist readily induces PKA phosphorylation of receptors, but fails to promote PKA phosphorylation of PLB at the SR and troponin I (TnI) on the myofibril (De Arcangelis et al., 2010). Additional inhibition of PDE4 significantly enhances PKA phosphorylation of PLB and TnI, suggesting that PDE4 plays a critical role in this segregation (De Arcangelis et al., 2010). To allow cAMP diffusion from the PM, one has to effectively remove/"inhibit" the $\beta A R$-associated PDE4 to enable the synthesized cAMP to diffuse into intracellular space. At high concentration of catecholamines, activation of $\beta$ ARs promotes robust PKA phosphorylation of PLB and TnI in cardiomyocytes, indicating that high concentrations of agonists trigger a mechanism to remove/"inhibit" PDE4D from the receptor/AC complexes or to out pace the cAMP hydrolysis by PDE4. Accordingly, high concentrations of agonists promote dissociation of PDE4D8 from $\beta_{1}$ AR in cardiomyocytes (De Arcangelis et al., 2010; Richter et al., 2008). Interestingly, stimulation of $\beta_{2} \mathrm{AR}$ with high concentration of agonist induces recruitment of PDE4D5 and 8 isoforms to $\beta_{2}$ receptor (De Arcangelis et al., 2009). This is due to that stimulation of $\beta_{2} \mathrm{AR}$ promotes receptor palmitoylation and phosphorylation by G-protein receptor kinase, which enhances recruitment of arrestin and PDE4D isoforms (Liu et al., 2012a). The arrestin binding also promotes receptor internalization; therefore, the internalized $\beta_{2} \mathrm{AR} /$ arrestin complexes may functionally sequestrate PDE4D5 and 8 from the PM. While recent reports indicate that GPCRs activated by peptide ligands can induce persistent cAMP signal even after internalization (Calebiro et al., 2010), it is remains to be addressed whether the internalized $\beta_{2} \mathrm{AR}$ can still induce cAMP on the endosomes. In comparison, $\beta_{1} \mathrm{AR}$, the major subtype for cAMP production in cardiomyocytes undergoes minimal internalization after agonist stimulation (Xiang et al., 2002). In this model, the internalized $\beta_{2} \mathrm{AR}$ may facilitate 
cAMP diffusion from the $\beta$ ARs on the PM to promote PKA-dependent cardiac contractile responses in both health and diseased hearts (Figure 1B).

After arriving at the SR, cAMP signals have to overcome other hurdles to activate PKA anchored by AKAPs on the intracellular organelles. This is because that PDEs localized on the SR can readily degrade the arriving cAMP molecules. Both PDE3 and PDE4 families are involved in maintaining the basal cAMP levels on the SR (Soto et al., 2009). Moreover, mAKAP anchors PKA together with PDE4D3 and PP2A to the RyR2 complex at the SR, and coordinates PKA phosphorylation of the ion channel (Dodge et al., 2001). To maximally enhance calcium release for contractile responses, the RyR2-associated PDE4D3 and PP2A need to be removed/"inhibited" to promote PKA phosphorylation of channel. Disruption of mAKAP complexes leads to aberrant calcium cycling associated with arrhythmia (Lehnart et al., 2005), likely due to dysregulation of cAMP/PKA activities at the RyR2 complexes. PDE4D is also associated with SERCA2 complexes. Dephosphorylated PLB is a high affinity inhibitor of SERCA2 for reuptake of $\mathrm{Ca}^{2+}$ into the SR; and phosphorylation of PLB during $\beta$-adrenergic stimulation relieves its inhibitory effects on SERCA2. Deletion of PDE4D promotes higher baseline levels of PKA phosphorylation of PLB and increased $\mathrm{Ca}^{2+}$ re-uptake into the SR for calcium signaling and contractile responses (Beca et al., 2011). Meanwhile, AKAP18 $\delta$ is reported to regulate PKA-mediated phosphorylation of PLB and increases the $\mathrm{Ca}^{2+}$ re-uptake into the SR (Lygren et al., 2007). Alteration in the localized PKA phosphorylation of SR proteins is believed to contribute to contractile dysfunction in heart failure. Therefore, mechanisms are adopted to safeguard random diffusion of cAMP from the receptor/Gs/AC complexes, and to promote the cAMP accumulation/distribution in these specific subcellular organelles.

Sarcomere represents another essential organelle targeted by the $\beta A R$-induced cAMP signal for contractile responses. AKAPs are also identified to anchor PKA in sarcomere for phosphorylation of myofibril proteins for contractile responses (Perino et al., 2012). Cardiac troponin $\mathrm{T}$ has been recently characterized as a novel dual-specificity AKAP that is able to dock PKA at the thin filaments in proximity of its sarcomeric substrates (Sumandea et al., 2011) to phosphorylate cardiac $\mathrm{TnI}$ and cardiac myosin binding protein $\mathrm{C}$ (cMyBPC) to enhance cardiac contractility. Stimulation of $\beta A R$ induces translocation of PP2A from the myofibril to the cytoplasm, and promotes PKA phosphorylation of cMyBPC in cardiomyocytes (Yin et al., 2010). Meanwhile, a dual AKAP myomegalin (MMGL) also assembles a $\mathrm{cAMP} / \mathrm{PKA} / \mathrm{PDE}$ signaling module at the sarcomere. $\beta \mathrm{AR}$ stimulation induces translocation of MMGL to the sarcomere and promotes PKA phosphorylation of cMyBPC and TnI to enhance cardiac contraction (Uys et al., 2011). Synemin is the first intermediate filament protein shown to bind PKA and to localize a pool of PKA to phosphorylate substrates at the Z-line (Russell et al., 2006). Whether dysfunction of cAMP/PKA activities on the sarcomere is involved in cardiac diseases remains poorly understood.

Last, many studies have shown that $\beta$-adrenergic stimulation induces activation of $\mathrm{Ca}^{2+} /$ calmodulin $\left(\mathrm{Ca}^{2+} / \mathrm{CaM}\right)$-dependent kinase II (CaMKII), and CaMKII-dependent phosphorylation of PLB and RyR (Swaminathan et al., 2012). The phosphorylation of PLB and RyR2 by CaMKII are distinct from those by PKA. However, it has been reported that CaMKII inhibition does not affect normal cardiac $\beta$-adrenergic responses (Zhang et al., 
2005). Therefore, whether $\beta A R$-induced CaMKII activities participate in the "fight or flight" response remains inconclusive. Nevertheless, chronic $\beta A R$-induced CaMKII activity in stressed hearts has a pronounced role in disease development (Anderson et al., 2011). Among $\beta \mathrm{ARs}, \beta_{1} \mathrm{AR}$ is shown to promote CaMKII activation whereas $\beta_{2} \mathrm{AR}$ does not. Studies have proposed that PKA activation is the prerequisite of CaMKII activation induced by $\beta A R$ stimulation (Ferrero et al., 2007). This is because PKA phosphorylates and enhances the local activities of LTCC and RyR2 (Soltis and Saucerman, 2010), which provides the sources of $\mathrm{Ca}^{2+}$ for CaMKII activation. These results also suggest that the $\mathrm{Ca}^{2+}$ signal for CaMKII activation after $\beta$ AR stimulation is probably via PKA-dependent local increases in $\mathrm{Ca}^{2+}$ concentration (Ferrero et al., 2007). Conversely, studies have also proposed PKAindependent mechanisms for CaMKII activation induced by $\beta A R$ agonists including Gsmediated direct upregulation of LTCC activity (Lader et al., 1998) and cAMP-dependent EPAC (exchange protein directly activated by cAMP)-mediated signaling pathway (Oestreich et al., 2009). The different pathways may underlie the possible activation of different pools of CaMKII under acute versus chronic adrenergic stimulation, and their roles in cardiac disease development.

\section{Spatial regulation of cAMP signals under multiple neurohormonal stimuli}

Consistent with its essential role as one of the major neurohormonal regulatory systems for cardiac contraction, these $\beta A R$ signal domains appear to be exclusive for adrenergic regulation in cardiomyocytes. The cAMP signals induced by other GPCRs including EP receptor, adenosine receptor and dopamine receptor display minimal to partial access to PKA involved in phosphorylation of PLB (Liu et al., 2012b). For example, stimulation of prostaglandin E receptors EP2 and EP4 induces robust cAMP signals in cardiomyocytes; but the cAMP signals fail to promote PKA phosphorylation of LTCCs (Agarwal et al., 2011). Studies show that the cAMP signals induced by EP receptors primarily activate type I PKA (Di Benedetto et al., 2008), but cannot communicate directly with type II PKA in the compartments for LTCCs and PLB (Agarwal et al., 2011). The difference between $\beta A R$ and other neurohormone-activated GPCR signaling pathways is not well understood.

Meanwhile, emerging evidence suggests that under different neurohormonal stimuli, the cAMP signals can be integrated intracellularly for a coordinated myocyte contractile responses (Liu et al., 2012b). Cross talk can take place at receptor/G protein/AC axis (Warrier et al., 2005) or at intracellular cAMP levels (Liu et al., 2012b). In the $\beta A R / G$ protein/AC cascade, $\mathrm{AC}$ acts as a rate-limiting factor due to its low copies of expression and catalytic activities (Gao et al., 1998). Thus, many receptors are able to couple to the same $G$ proteins; and different $\mathrm{G}$ proteins can regulate the same ACs for cAMP synthesis. For example, activation of muscarinic receptor couples to $\mathrm{Gi}$, and attenuates the local $\beta \mathrm{AR}$ induced cAMP signals in caveoli for contractile responses (Warrier et al., 2005). However, the potent inhibitory effect of muscarinic receptor on the $\beta A R$-induced cAMP signals raises the possibilities that other mechanisms are also involved in this cross talk.

Recently, cross talk has been characterized at the level of cAMP degradation between adrenergic and other Gs-coupled receptor signaling cascades (Figure 1C). In this case, activation of EP4 promotes a cAMP signal sufficient to activate type I PKA in 
cardiomyocytes (Di Benedetto et al., 2008). One of targets by this PKA activity is the PDE4D in association with $\beta$ ARs (Liu et al., 2012b). EP4 activation also blocks dissociation of PDE4D isoforms from the activated $\beta$ ARs. Thus, under EP4 activation, the activated PDE4D isoforms in the $\beta A R$ complexes readily hydrolyze the cAMP induced by adrenergic stimulation, and effectively prevent cAMP diffusion for PKA phosphorylation of PLB and contractile responses (Liu et al., 2012b). Several other neurohormonal stimuli behave similarly in cross talking with the $\beta$ AR pathway, suggesting a general mechanism for integration of receptor signaling intracellularly (Liu et al., 2012b). Understanding the impacts of these neurohormonal regulation of $\beta A R$-induced cAMP signals in cardiomyocytes can provide critical and novel insights of development of cardiac diseases such as hypertrophy and heart failure.

Moreover, PDE2 coordinates cGMP and cAMP signaling in another feedback control loop of $\beta$-adrenergic pathway (Stangherlin and Zaccolo, 2012). In this case, cGMP signals activated by NO pathways are sufficient to cross-inhibit cAMP signals induced by $\beta A R$ (Stangherlin and Zaccolo, 2012). Moreover, stimulation of $\beta$ AR leads to activation of the NO-cGMP-PDE2 pathway, which counteracts cAMP signals obtained via stimulation of $\beta_{1}$ and $\beta_{2}$ ARs (Mongillo et al., 2006). Meanwhile, PDE5 can inhibits a $\beta$ AR-dependent cascade for protein kinase G-dependent phosphorylation of TnI, and inhibit sarcomere shortening without altering calcium transient (Lee et al., 2010). This is due to local effects of $\beta$ AR-cGMP compartments controlled by PDE5, which are distinct from those cGMP compartments accessible to PDE2 and PDE3 (Lee et al., 2010). Selective targeting different pools of cAMP and cGMP may offer benefit in cardiac therapy.

\section{Spatiotemporal $\beta$ AR signals in heart failure}

Uncontrolled cellular cAMP signals can potentially lead to alteration of cellular homeostasis by inducing either adaptive gene expression or cell death. During development of heart failure, increased sympathetic activities drive the $\beta$ AR stimulation in cardiomyocytes, promoting higher intracellular cAMP signals for compensatory cardiac function in the heart. However, persistent cAMP signals are dangerous since they can activate other cellular proteins for either maladaptive or detrimental cellular responses such as cell apoptosis (Zhu et al., 2003). Consequently, the expression levels of $\beta_{1} \mathrm{AR}$ are reduced to attenuate stimulation of cAMP synthesis in cardiomyocytes (Nikolaev et al., 2010). Meanwhile, the expression of Gi proteins is increased, which may also serve to curtail excessive intracellular cAMP signals under elevated sympathetic-adrenergic drive (Eschenhagen et al., 1992). In comparison, the expression of $\beta_{2} \mathrm{AR}$ is not altered. The altered ratio between $\beta_{1} \mathrm{AR}$ and $\beta_{2} \mathrm{AR}$ converts the latter to be the major $\beta \mathrm{AR}$ subtype in failing hearts. Interestingly, $\beta_{2} \mathrm{AR}$ behaves like $\beta_{1} \mathrm{AR}$ in the failing cardiomyocytes, and the $\beta_{2} \mathrm{AR}$-induced cAMP signals is increased and broadly distributed in the cell (Nikolaev et al., 2010). The implication of these broadly distributed cAMP signals in modulating contractile properties in failing hearts are yet to be studied.

The expression of PDE4 as well as many other PDE isoforms has been elevated during adaptive myocardium hypertrophy (Masunaga et al., 2004). The increased PDE expression may also serve as a protective mechanism that curtails the cAMP levels and prevents 
unwanted PKA activities in cardiomyocytes. Selective inhibition of PDEs could be explored to enhance contractile responses without other detrimental phosphorylation events. In comparison, much less is known about the expression and activities of PDE families during the decompensatory heart failure. One notable example is the decreased association of PDE4D3 with RyR2 complex on the SR, which leads to higher basal phosphorylation of RyR2 and calcium release (calcium leaking), contributing to increased cardiac arrhythmia in heart failure (Lehnart et al., 2005). In this case, restoring the location and function of PDE4D3 rather than inhibition of the enzyme may be a therapeutic strategy to reduce cardiac arrhythmia in heart failure.

Chronic $\beta$ AR stimulation also promotes CaMKII-dependent contractile responses. In failing cardiomyocytes, $\beta_{2} \mathrm{AR}$ mimics $\beta_{1} \mathrm{AR}$, and is capable of activating CaMKII (Anderson et al., 2011). Chronic $\beta A R$-induced CaMKII activity in stressed hearts has a pronounced role in promoting the development of cardiac hypertrophy, myocyte apoptosis, and cardiac dysfunction and arrhythmias by causing SR Ca ${ }^{2+}$ overload (Anderson et al., 2011). Inhibition of CaMKII is able to ameliorate cardiac remodeling and reduce cardiac arrhythmias after myocardial infarction (Zhang et al., 2005). Although the direct link is still missing, the detrimental CaMKII activities in cardiomyocytes could be related to dysregulation of distribution of cAMP signals under chronic $\beta$ AR stimulation.

\section{Perspectives}

The concept of compartmentalization of cAMP signals has been proposed for more than 30 years. Only after availability of a series of genetically coded biosensors in the past 10 years to detect second messengers and kinase activities in live cells, we began to understand and appreciate dynamics of the compartmentalized $\beta$ AR signals. Many chronic conditions such as inflammation may induce subtle changes of compartmentalized $\beta A R$ signals in cardiomyocytes (Liu et al., 2012b). These changes can potentially enhance intracellular cAMP signals or inhibit the propagation of cAMP into distinct cellular compartments. While the former could lead to spillover of cAMP outside the confined $\beta$ AR signaling domains and generate unwanted toxic cellular signals, the latter could trigger compensatory elevation of sympathetic activities to chronically stimulate $\beta$ ARs. These alterations in compartmentalized $\beta A R$ signals may contribute directly and independently to onset as well as progression of heart failure. Understanding mechanisms that govern compartmentalized $\beta$ AR signals may provide new paradigms on heart failure development and novel therapeutic strategies for treating the diseases.

\section{References}

Agarwal SR, MacDougall DA, Tyser R, Pugh SD, Calaghan SC, Harvey RD. Effects of cholesterol depletion on compartmentalized cAMP responses in adult cardiac myocytes. J Mol Cell Cardiol. 2011; 50:500-509. [PubMed: 21115018]

Anderson ME, Brown JH, Bers DM. CaMKII in myocardial hypertrophy and heart failure. J Mol Cell Cardiol. 2011; 51:468-473. [PubMed: 21276796]

Balijepalli RC, Foell JD, Hall DD, Hell JW, Kamp TJ. Localization of cardiac L-type Ca(2+) channels to a caveolar macromolecular signaling complex is required for beta(2)-adrenergic regulation. Proc Natl Acad Sci U S A. 2006; 103:7500-7505. [PubMed: 16648270] 
Barbuti A, Terragni B, Brioschi C, DiFrancesco D. Localization of $\mathrm{f}$-channels to caveolae mediates specific beta2-adrenergic receptor modulation of rate in sinoatrial myocytes. J Mol Cell Cardiol. 2007; 42:71-78. [PubMed: 17070839]

Beca S, Helli PB, Simpson JA, et al. Phosphodiesterase 4D regulates baseline sarcoplasmic reticulum $\mathrm{Ca} 2+$ release and cardiac contractility, independently of L-type $\mathrm{Ca} 2+$ current. Circulation research. 2011; 109:1024-1030. [PubMed: 21903937]

Calebiro D, Nikolaev VO, Persani L, Lohse MJ. Signaling by internalized G-protein-coupled receptors. Trends Pharmacol Sci. 2010; 31:221-228. [PubMed: 20303186]

De Arcangelis V, Liu R, Soto D, Xiang Y. Differential association of phosphodiesterase 4D isoforms with beta2-adrenoceptor in cardiac myocytes. J Biol Chem. 2009; 284:33824-33832. [PubMed: 19801680]

De Arcangelis V, Liu S, Zhang D, Soto D, Xiang YK. Equilibrium between adenylyl cyclase and phosphodiesterase patterns adrenergic agonist dose-dependent spatiotemporal cAMP/protein kinase A activities in cardiomyocytes. Mol Pharmacol. 2010; 78:340-349. [PubMed: 20530128]

De Arcangelis V, Soto D, Xiang Y. Phosphodiesterase 4 and phosphatase 2A differentially regulate cAMP/protein kinase a signaling for cardiac myocyte contraction under stimulation of beta1 adrenergic receptor. Mol Pharmacol. 2008; 74:1453-1462. [PubMed: 18703669]

Di Benedetto G, Zoccarato A, Lissandron V, et al. Protein kinase A type I and type II define distinct intracellular signaling compartments. Circulation research. 2008; 103:836-844. [PubMed: 18757829]

Dodge KL, Khouangsathiene S, Kapiloff MS, et al. mAKAP assembles a protein kinase A/PDE4 phosphodiesterase cAMP signaling module. Embo J. 2001; 20:1921-1930. [PubMed: 11296225]

Dodge-Kafka KL, Bauman A, Mayer N, et al. cAMP-stimulated protein phosphatase 2A activity associated with muscle A kinase-anchoring protein (mAKAP) signaling complexes inhibits the phosphorylation and activity of the cAMP-specific phosphodiesterase PDE4D3. J Biol Chem. 2010; 285:11078-11086. [PubMed: 20106966]

Eschenhagen T, Mende U, Nose M, et al. Increased messenger RNA level of the inhibitory G protein alpha subunit Gi alpha-2 in human end-stage heart failure. Circulation research. 1992; 70:688696. [PubMed: 1551195]

Ferrero P, Said M, Sanchez G, et al. Ca2+/calmodulin kinase II increases ryanodine binding and Ca2+induced sarcoplasmic reticulum $\mathrm{Ca} 2+$ release kinetics during beta-adrenergic stimulation. J Mol Cell Cardiol. 2007; 43:281-291. [PubMed: 17643448]

Gao M, Ping P, Post S, Insel PA, Tang R, Hammond HK. Increased expression of adenylylcyclase type VI proportionately increases $\beta$-adrenergic receptor-stimulated production of cAMP in neonatal rat cardiac myocytes. Proc Natl Acad Sci U S A. 1998; 95:1038-1043. [PubMed: 9448281]

Lader AS, Xiao YF, Ishikawa Y, et al. Cardiac Gsalpha overexpression enhances L-type calcium channels through an adenylyl cyclase independent pathway. Proc Natl Acad Sci U S A. 1998; 95:9669-9674. [PubMed: 9689139]

Lee DI, Vahebi S, Tocchetti CG, et al. PDE5A suppression of acute beta-adrenergic activation requires modulation of myocyte beta-3 signaling coupled to PKG-mediated troponin I phosphorylation. Basic Res Cardiol. 2010; 105:337-347. [PubMed: 20107996]

Lefkowitz RJ. Seven transmembrane receptors: something old, something new. Acta Physiol (Oxf). 2007; 190:9-19. [PubMed: 17428228]

Lehnart SE, Wehrens XH, Reiken S, et al. Phosphodiesterase 4D deficiency in the ryanodine-receptor complex promotes heart failure and arrhythmias. Cell. 2005; 123:25-35. [PubMed: 16213210]

Liu R, Wang D, Shi Q, Fu Q, Hizon S, Xiang YK. Palmitoylation regulates intracellular trafficking of beta2 adrenergic receptor/arrestin/phosphodiesterase 4D complexes in cardiomyocytes. PLoS One. 2012a; 7:e42658. [PubMed: 22912718]

Liu S, Li Y, Kim S, et al. Phosphodiesterases coordinate cAMP propagation induced by two stimulatory G protein-coupled receptors in hearts. Proc Natl Acad Sci U S A. 2012b; 109:65786583. [PubMed: 22493261]

Lygren B, Carlson CR, Santamaria K, et al. AKAP complex regulates Ca2+ re-uptake into heart sarcoplasmic reticulum. EMBO Rep. 2007; 8:1061-1067. [PubMed: 17901878] 
Macdougall DA, Agarwal SR, Stopford EA, et al. Caveolae compartmentalise beta2-adrenoceptor signals by curtailing cAMP production and maintaining phosphatase activity in the sarcoplasmic reticulum of the adult ventricular myocyte. J Mol Cell Cardiol. 2012; 52:388-400. [PubMed: 21740911]

Masunaga R, Nagasaka A, Sawai Y, et al. Changes in cyclic nucleotide phosphodiesterase activity and calmodulin concentration in heart muscle of cardiomyopathic hamsters. J Mol Cell Cardiol. 2004; 37:767-774. [PubMed: 15350849]

Mongillo M, McSorley T, Evellin S, et al. Fluorescence resonance energy transfer-based analysis of cAMP dynamics in live neonatal rat cardiac myocytes reveals distinct functions of compartmentalized phosphodiesterases. Circ Res. 2004; 95:67-75. [PubMed: 15178638]

Mongillo M, Tocchetti CG, Terrin A, et al. Compartmentalized phosphodiesterase-2 activity blunts beta-adrenergic cardiac inotropy via an NO/cGMP-dependent pathway. Circ Res. 2006; 98:226234. [PubMed: 16357307]

Nichols CB, Rossow CF, Navedo MF, et al. Sympathetic stimulation of adult cardiomyocytes requires association of AKAP5 with a subpopulation of L-type calcium channels. Circulation research. 2010; 107:747-756. [PubMed: 20671242]

Nikolaev VO, Bunemann M, Schmitteckert E, Lohse MJ, Engelhardt S. Cyclic AMP imaging in adult cardiac myocytes reveals far-reaching beta1-adrenergic but locally confined beta2-adrenergic receptor-mediated signaling. Circ Res. 2006; 99:1084-1091. [PubMed: 17038640]

Nikolaev VO, Moshkov A, Lyon AR, et al. Beta2-adrenergic receptor redistribution in heart failure changes cAMP compartmentation. Science. 2010; 327:1653-1657. [PubMed: 20185685]

Oestreich EA, Malik S, Goonasekera SA, et al. Epac and phospholipase Cepsilon regulate Ca2+ release in the heart by activation of protein kinase Cepsilon and calcium-calmodulin kinase II. J Biol Chem. 2009; 284:1514-1522. [PubMed: 18957419]

Okumura S, Kawabe J, Yatani A, et al. Type 5 adenylyl cyclase disruption alters not only sympathetic but also parasympathetic and calcium-mediated cardiac regulation. Circulation research. 2003; 93:364-371. [PubMed: 12869393]

Perino A, Ghigo A, Scott JD, Hirsch E. Anchoring proteins as regulators of signaling pathways. Circulation research. 2012; 111:482-492. [PubMed: 22859670]

Richter W, Day P, Agrawal R, et al. Signaling from beta1- and beta2-adrenergic receptors is defined by differential interactions with PDE4. Embo J. 2008; 27:384-393. [PubMed: 18188154]

Richter W, Xie M, Scheitrum C, Krall J, Movsesian MA, Conti M. Conserved expression and functions of PDE4 in rodent and human heart. Basic Res Cardiol. 2011; 106:249-262. [PubMed: 21161247]

Russel FG, Koenderink JB, Masereeuw R. Multidrug resistance protein 4 (MRP4/ABCC4): a versatile efflux transporter for drugs and signalling molecules. Trends Pharmacol Sci. 2008; 29:200-207. [PubMed: 18353444]

Russell MA, Lund LM, Haber R, McKeegan K, Cianciola N, Bond M. The intermediate filament protein, synemin, is an AKAP in the heart. Arch Biochem Biophys. 2006; 456:204-215. [PubMed: 16934740]

Sellers ZM, Naren AP, Xiang Y, Best PM. MRP4 and CFTR in the regulation of cAMP and betaadrenergic contraction in cardiac myocytes. Eur J Pharmacol. 2012; 681:80-87. [PubMed: 22381067]

Sette C, Conti M. Phosphorylation and activation of a cAMP-specific phosphodiesterase by the cAMPdependent protein kinase. Involvement of serine 54 in the enzyme activation. J Biol Chem. 1996; 271:16526-16534. [PubMed: 8663227]

Soltis AR, Saucerman JJ. Synergy between CaMKII substrates and beta-adrenergic signaling in regulation of cardiac myocyte $\mathrm{Ca}(2+)$ handling. Biophysical journal. 2010; 99:2038-2047. [PubMed: 20923637]

Soto D, De Arcangelis V, Zhang J, Xiang Y. Dynamic protein kinase a activities induced by betaadrenoceptors dictate signaling propagation for substrate phosphorylation and myocyte contraction. Circ Res. 2009; 104:770-779. [PubMed: 19213958]

Stangherlin A, Zaccolo M. cGMP-cAMP interplay in cardiac myocytes: a local affair with far-reaching consequences for heart function. Biochem Soc Trans. 2012; 40:11-14. [PubMed: 22260658] 
Sumandea CA, Garcia-Cazarin ML, Bozio CH, Sievert GA, Balke CW, Sumandea MP. Cardiac troponin T, a sarcomeric AKAP, tethers protein kinase A at the myofilaments. The Journal of biological chemistry. 2011; 286:530-541. [PubMed: 21056973]

Swaminathan PD, Purohit A, Hund TJ, Anderson ME. Calmodulin-dependent protein kinase II: linking heart failure and arrhythmias. Circ Res. 2012; 110:1661-1677. [PubMed: 22679140]

Tao J, Malbon CC. G-protein-coupled receptor-associated A-kinase anchoring proteins AKAP5 and AKAP12: differential signaling to MAPK and GPCR recycling. J Mol Signal. 2008; 3:19. [PubMed: 19055733]

Uys GM, Ramburan A, Loos B, et al. Myomegalin is a novel A-kinase anchoring protein involved in the phosphorylation of cardiac myosin binding protein C. BMC Cell Biol. 2011; 12:18. [PubMed: 21569246]

Warrier S, Belevych AE, Ruse M, et al. Beta-adrenergic- and muscarinic receptor-induced changes in cAMP activity in adult cardiac myocytes detected with FRET-based biosensor. Am J Physiol Cell Physiol. 2005; 289:C455-461. [PubMed: 15788489]

Willoughby D, Wong W, Schaack J, Scott JD, Cooper DM. An anchored PKA and PDE4 complex regulates subplasmalemmal cAMP dynamics. Embo J. 2006; 25:2051-2061. [PubMed: 16642035]

Xiang Y, Devic E, Kobilka B. The PDZ binding motif of the Beta 1 adrenergic receptor modulates receptor trafficking and signaling in cardiac myocytes. J Biol Chem. 2002; 277:33783-33790. [PubMed: 12097326]

Xiang Y, Naro F, Zoudilova M, Jin SL, Conti M, Kobilka B. Phosphodiesterase 4D is required for \{beta\}2 adrenoceptor subtype-specific signaling in cardiac myocytes. Proc Natl Acad Sci U S A. 2005

Xiang YK. Compartmentalization of beta-adrenergic signals in cardiomyocytes. Circ Res. 2011; 109:231-244. [PubMed: 21737818]

Xiao RP, Hohl C, Altschuld R, et al. Beta 2-adrenergic receptor-stimulated increase in cAMP in rat heart cells is not coupled to changes in $\mathrm{Ca} 2+$ dynamics, contractility, or phospholamban phosphorylation. J Biol Chem. 1994; 269:19151-19156. [PubMed: 8034672]

Yin X, Cuello F, Mayr U, et al. Proteomics analysis of the cardiac myofilament subproteome reveals dynamic alterations in phosphatase subunit distribution. Mol Cell Proteomics. 2010; 9:497-509. [PubMed: 20037178]

Zaccolo M. Phosphodiesterases and compartmentalized cAMP signalling in the heart. Eur J Cell Biol. 2006; 85:693-697. [PubMed: 16466668]

Zaccolo M, Pozzan T. Discrete microdomains with high concentration of cAMP in stimulated rat neonatal cardiac myocytes. Science. 2002; 295:1711-1715. [PubMed: 11872839]

Zhang R, Khoo MS, Wu Y, et al. Calmodulin kinase II inhibition protects against structural heart disease. Nature medicine. 2005; 11:409-417.

Zhu WZ, Wang SQ, Chakir K, et al. Linkage of beta1-adrenergic stimulation to apoptotic heart cell death through protein kinase A-independent activation of Ca2+/calmodulin kinase II. J Clin Invest. 2003; 111:617-625. [PubMed: 12618516] 
A

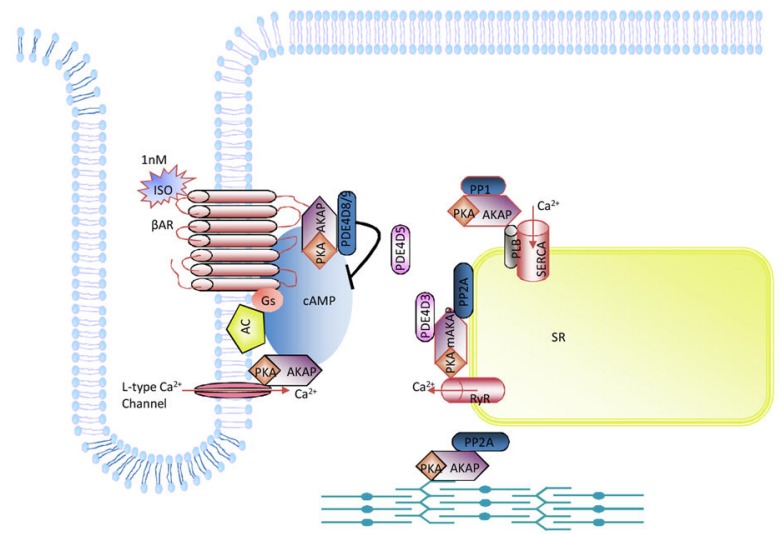

B

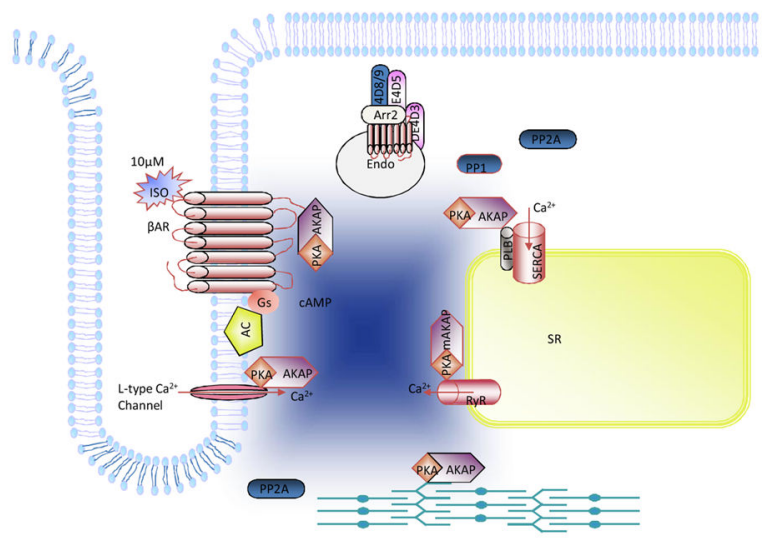

C

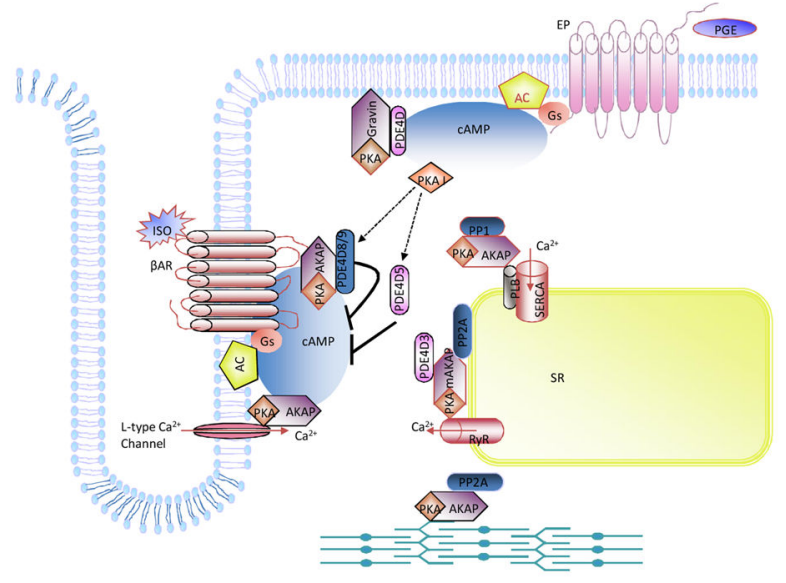

Figure 1.

Model of compartmentalized $\beta$-adrenergic signals in cardiomyocytes. A. At $10^{-9} \mathrm{M}$ isoproterenol, Activation of $\beta$ AR leads to stimulation of AC to produce cAMP. The cAMP signals promote activation of PKA that associates with receptor via AKAPs. The activated PKA phosphorylates the receptor and receptor-associated PDE that negatively feeds back to attenuate the cAMP signals. As a result, the cAMP signals are transient and restricted at the vicinity of the receptor. $\mathrm{B}$. At $10^{-5} \mathrm{M}$ isoproterenol, besides the cAMP production via the $\beta \mathrm{AR} / \mathrm{AC}$ axis, the activated $\beta \mathrm{AR}$ also undergo arrestin-mediated receptor internalization. 
Meanwhile, the PDE4D isoforms are dissociated from the receptor at the PM, and sequestrated by the internalized receptor/arrestin complexes on endosomes. Therefore, the cAMP can diffuse and propagate to access PKA in different subcellular organelles. Moreover, the PDEs and PPs at the SR and myofibril may also dissociate from the compartment to promote local cAMP signals for PKA activation and phosphorylation of substrates. As a result, the activated PKA can phosphorylate both local (near the receptor) and distant substrates to enhance myocyte contractile responses. C. Activation of EPs induces cAMP signals at local membrane domains, which promotes activation of type I PKA. The activated PKA can phosphorylate PDE4D isoforms, including the $\beta A R$-associated PDE4D isoforms, which prevents the $\beta A R$-induced cAMP propagation from the PM domain to the SR membrane. Thus, activation of EPs impairs the intracellular $\beta$ AR signaling transduction and inhibits the $\beta$ AR-induced PKA phosphorylation and myocyte contractile responses. Endo, endosome; Iso, isoproterenol. 